\title{
Analyst
}
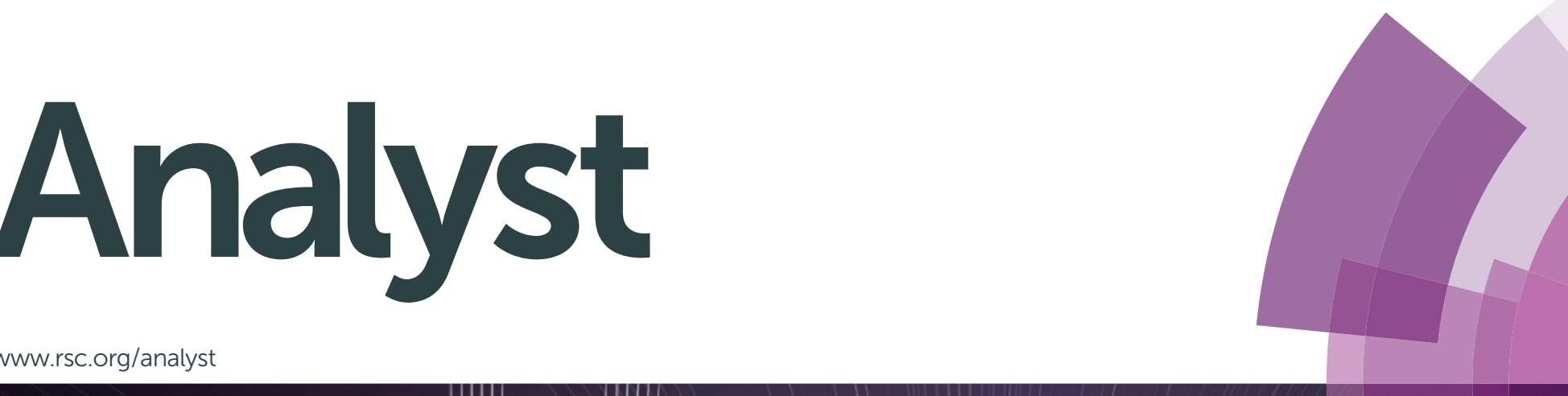


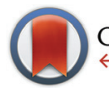

CrossMark

\&lick for updates

Cite this: Analyst, 2016, 141, 6202

Received 17th May 2016,

Accepted 7th August 2016

DOI: 10.1039/c6an01130f

www.rsc.org/analyst

\section{Advanced gas sensors based on substrate- integrated hollow waveguides and dual-color ring quantum cascade lasers}

\author{
Erhan Tütüncü, ${ }^{a}$ Vjekoslav Kokoric, ${ }^{a}$ Rolf Szedlak, ${ }^{\mathrm{b}}$ Donald MacFarland, ${ }^{\mathrm{b}}$ \\ Tobias Zederbauer, ${ }^{c}$ Hermann Detz, ${ }^{d}$ Aaron Maxwell Andrews, ${ }^{b}$ Werner Schrenk, ${ }^{c}$ \\ Gottfried Strasser ${ }^{\mathrm{b}, \mathrm{c}}$ and Boris Mizaikoff*a
}

This study shows the first combination of a ring-shaped vertically emitting quantum cascade laser (riQCL) providing two distinct emission wavelengths combined with a substrate-integrated hollow waveguide (iHWG). This ultra-compact riQCL-iHWG gas sensing device enables the simultaneous detection of two vapor phase species - here, furan and 2-methoxyethanol - providing distinctive absorption features at the emission wavelengths of the riQCL (i.e., 1144 and $1170 \mathrm{~cm}^{-1}$ ). Hence, multianalyte gas sensing via a unique mid-infrared (MIR) sensor concept is demonstrated.

\section{Introduction}

Infrared spectroscopy, and in particular the MIR spectral range (2-20 $\mu \mathrm{m})$ provides a viable alternative to commonly used vapor phase analysis techniques (e.g., gas chromatography combined with various detection schemes, mass spectrometric techniques, etc.) for selectively, sensitively, and rapidly detecting or monitoring molecular constituents. Due to the substantial potential for miniaturization and given recent progress in IR waveguide, light source, and detector technology ${ }^{1-3}$ along with inherent molecular selectivity and minimal sample preparation, conventional infrared absorption spectroscopy has evolved into a versatile and modular sensing toolbox. ${ }^{4}$

The excitation of fundamental vibro-rotational transitions is usually initiated via either broadband light sources such as incandescent glow bars, or using narrowband emitters including initially lead salt laser diodes, and nowadays predominantly quantum cascade lasers (QCLs) and interband cascade lasers (ICLs). Clearly, MIR sensing applications benefit from a possibly broad wavelength coverage, if entire molecular spectra or the discrimination of a multitude of analytes with poten-

\footnotetext{
${ }^{a}$ Institute of Analytical and Bioanalytical Chemistry, Ulm University, Germany. E-mail: boris.mizaikoff@uni-ulm.de

${ }^{b}$ Institute of Solid State Electronics, TU Wien, Austria

${ }^{c}$ Center for Micro- and Nanostructures, TU Wien, Austria

${ }^{d}$ Austrian Academy of Sciences, Austria
}

tially overlapping spectral features is required. Alternatively, selected target analytes may be addressed via comparatively narrow spectral bands selecting a single yet analyte-specific vibro-rotational absorption line free from interferences with any other gas phase constituent present in the sampled scenario.

However, a variety of analytical applications demand for a compromise between these two scenarios (i.e., broadband vs. narrowband IR absorption spectroscopy) for addressing moderately complex samples containing a limited number of constituents, yet taking advantage of the spectral brightness and compactness of advanced laser light sources. This requirement may be met by either using tunable laser light sources, or lasers based on active gain media tailored to emit at two or more wavelengths. While the latter concept provides coverage of few selected wavelengths only, the advantages in terms of device complexity, laser control, compactness, robustness, and power consumption are immediately evident.

Quantum cascade lasers (QCL) were first reported in $1994,^{5}$ and offer nowadays access to almost any wavelength window in the MIR via engineering of the quantum heterostructure comprising the active gain medium. Efficient photon emission via intersubband transitions instead of electron-hole recombination prevalent in conventional heterostructure laser diodes has rendered QCLs the MIR light source of choice in a wide variety of state-of-the-art optical sensing and spectroscopy concepts. Thereby, one may directly capitalize on their compact dimensions, robustness, long life time, and superior emission characteristics. $^{6-8}$

Edge-emitting Fabry-Pérot (FP) resonators are the most common optical configuration applied in diode laser light sources with the facets of the active laser chip acting as cavity mirrors. Recently, the research group of Strasser has reported a new generation of surface emitting QCLs based on ringshaped active structures (riQCL; Fig. 1) utilizing a second order distributed feedback (DFB) grating fabricated into the ring waveguide for efficient vertical light outcoupling. ${ }^{9-11}$ Besides facilitating pronounced optical power ${ }^{12}$ and excellent 


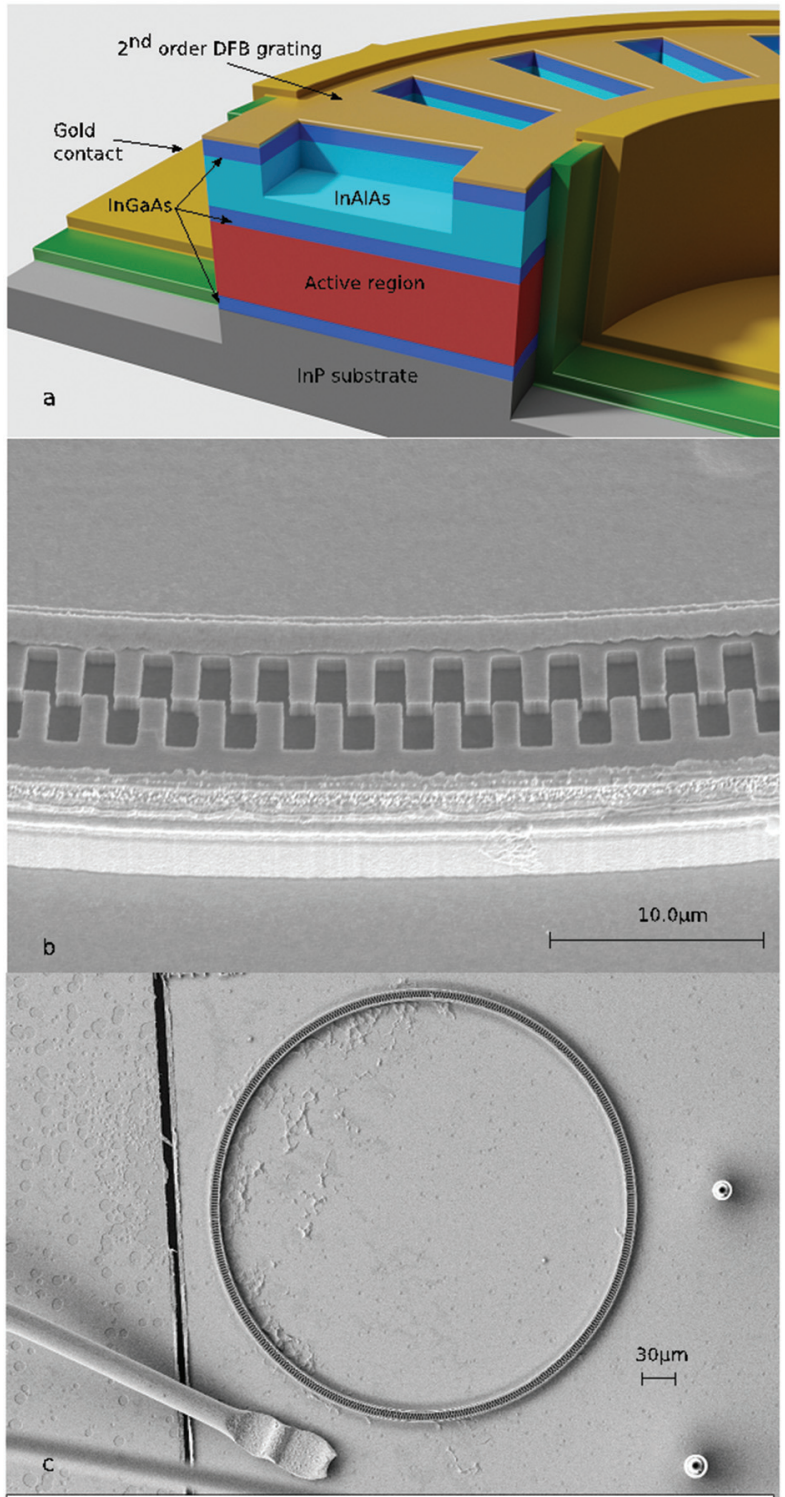

Fig. 1 (a) Schematic composition of a ring-shaped quantum cascade laser (riQCL) heterostructure and waveguide. The InGaAs/InAlAs active region is sandwiched between two InGaAs layers for improving vertical mode confinement. Electrical current is injected via an extended gold contact. The second order distributed feedback (DFB) grating on top of the waveguide selects the lasing wavelength and enables surface emission. (b, c) Scanning electron microscopy images of a riQCL structure with etched second-order gratings.

beam collimation, ${ }^{13-18}$ the integration of vertically emitting laser diodes facilitates their application within ultra-compact sensing concepts using a minimum of beam-guiding optical elements. From the perspective of optical efficiency, in addition a rotationally symmetric far field is achieved via dielectric meta-materials, thereby minimizing scattering losses.

Next to the light source, for the development of rapidly responding and portable gas analyzers miniaturized yet efficient gas cells are mandatory along with efficient coupling of the radiation emitted by the light source into the sample compartment. Conventional multipass gas cells are of limited utility in portable usage due to complex alignment, optical delicacy, and rather large required gas sample volumes. An innovative solution for highly miniaturized gas cells serving at the same time as efficient photon conduits has recently been presented by Mizaikoff and collaborators. These so-called substrate-integrated hollow waveguides (iHWGs) simultaneously serve as efficient optical waveguide and highly miniaturized gas cell. ${ }^{19-21}$ iHWGs enable addressing transient signals in minute gas sample volumes (i.e., few hundreds of microliters), and provide excellent signal-to-noise ratios (SNR) during absorption measurements. Hence, exquisite sensitivity along with ultra-fast sample transition times are achieved by the obtained so-called volumetric optical efficiency, which is superior to conventional long-path or multipass gas cells. Moreover, the design flexibility and mechanical robustness have proven iHWGs as a promising generic key component for a variety of infrared gas sensing scenarios including environmental analysis, process monitoring, and breath diagnostics. $^{22,23}$

The aim of the present study was demonstrating the first riQCL-iHWG prototype providing a compact yet high-performance gas/vapor mid-infrared sensor system comprising a low volume (i.e., $600 \mu \mathrm{L}$ ) iHWG gas cell combined with a multicolor riQCL enabling multi-component gas sensing.

\section{Experimental}

\section{Chemicals}

The vapor phase samples were prepared by collecting the head space gas above the corresponding liquid phase analytes. Furan (99\% stab. with 250 ppm butylhydroxytoluen Alfa Aeser, Germany) and 2-methoxyethanol (Sigma Aldrich, Germany) were transferred into serum vials. After sealing the vial with a serum cap, the vial containing 2-methoxyethanol was transferred into a $40{ }^{\circ} \mathrm{C}$ water bath at a depth slightly above the liquid level inside the vial for enhancing the vapour pressure. In contrast, the furan-containing vial was placed in an icebox at $0{ }^{\circ} \mathrm{C}$ during the experiments in order to correspondingly decrease the vapor phase.

Head space samples were then collected by inserting the needle of $50 \mathrm{~mL}$ plastic syringe (B. Braun Melsungen AG, Melsungen, Germany) through the cap into the vial. Gas mixtures 0 to $100 \%$ of each analyte were prepared via a static mixing procedure using two $50 \mathrm{~mL}$ plastic syringes ensuring thorough mixing. Each sample was analyzed three times.

\section{Substrate integrated hollow waveguide}

Radiation from the QCL was collimated into the iHWG gas cell, and propagated along the waveguiding channel. The iHWG was fabricated from brass with dimensions of $150 \times 25$ $\times 20 \mathrm{~mm}^{3}(L \times W \times H)$, and comprised a waveguiding channel with a cross-section of $2.0 \mathrm{~mm}^{2}$ simultaneously serving as miniaturized gas cell. As optimum waveguiding is achieved by 
high surface reflectivity, the top- and base-substrates were polished to a mirror-like finish using commercially available diamond polishing suspensions. Protection of brass from oxidation and for enhancing the adhesion between the substrate and the subsequently applied gold layer galvanic copper plating (copper film thickness approx. $1 \mu \mathrm{m}$ ) was applied. The gold layer was then galvanically deposited onto the $\mathrm{Cu}$-coated brass substrate for improving the reflectivity. Detailed information on the surface quality and assembly of iHWGs has been published in detail elsewhere. ${ }^{19,24,25}$

\section{Characterization of the riQCL}

The experimental sensor setup is schematically illustrated in Fig. 2. MIR radiation emitted from the riQCL (A) was collimated via a 1.5" ZnSe lens (B), and then guided via a goldcoated iHWG with a waveguide channel/gas cell length of $15 \mathrm{~cm}$. Radiation emanating at the distal end of the iHWG was directly illuminating a $4 \mathrm{~mm}^{2} \mathrm{HgCdTe}$ (MCT) detector element (FTIR-16-2.00 MSL-12, InfraRed Associates Inc., Stuart, FL, USA) located in a dewar cooled with liquid nitrogen (D). In a next step, it is anticipated that the riQCL and a thermoelectrically cooled MCT will be directly integrated into the iHWG structure, thus eliminating even the beam collimating ZnSe lens by coupling the emitted radiation instantaneously into the waveguide/gas cell channel.

Current pulses at the required repetition frequency were applied to the riQCL via a pulse generator (HP 8114A, Hewlett Packard, Houston USA). The riQCL was modulated at $10 \mathrm{kHz}$ at a pulse duration of either $250 \mathrm{~ns}$ or $100 \mathrm{~ns}$. The MCT detector output was coupled to a lock-in amplifier (Model 5209, Princeton Applied Research, NJ, USA) locked to the modulation frequency of the emitter. Data was recorded via the lockin amplifier, and simultaneously also directly from the MCT

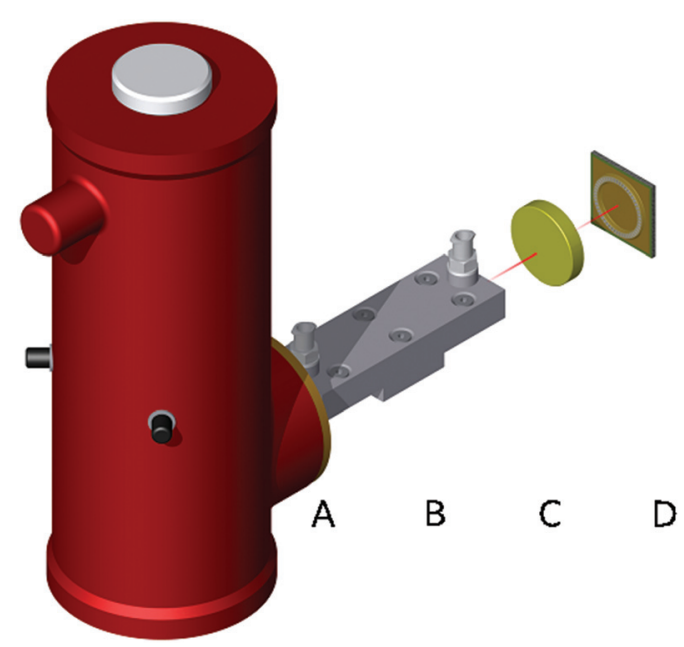

Fig. 2 Prototype sensing setup comprising a HgCdTe semiconductor detector (A), an iWHG with $15 \mathrm{~cm}$ waveguide/gas cell channel length (B), a ZnSe lens for beam collimation (C), and a riQCL (D). In a next step, it is anticipated that the riQCL and the detector will be directly integrated into the iHWG structure. (Dimensions are not to scale). detector. The latter voltage measurements were performed using a digital oscilloscope (PicoScope 5444B, Picotech, Cambridgeshire, UK). The riQCL features a dual DFB grating ${ }^{16}$ promoting/facilitating dual-color vertical emission of MIR radiation. The two distinct emission wavelengths at $1144 \mathrm{~cm}^{-1}$ $\left(5.5 \mathrm{kA} \mathrm{cm}^{-2}\right)$, and at $1170 \mathrm{~cm}^{-1}\left(10 \mathrm{kA} \mathrm{cm}^{-2}\right)$ are attributed to the $1^{\text {st }}$ and $2^{\text {nd }}$ order whispering gallery modes, respectively.

\section{FT-IR spectroscopy studies}

FT-IR measurements were performed for verification of the riQCL-iHWG sensor response to mixtures of furan and 2-methoxyethanol by focusing the external collimated IR beam provided by the spectrometer (Bruker Alpha, Bruker Optics, Ettlingen, Germany) onto the end facet of an iHWG $(7.5 \mathrm{~cm}$ waveguide/gas cell channel length; $300 \mu \mathrm{L}$ internal volume) using a gold-coated off-axis parabolic mirror (OAPM; focal length: $25.4 \mathrm{~mm}$ ). IR radiation emanating at the distal end of the waveguide was focused onto the DTGS detector integrated into the FT-IR spectrometer.

In between each measurement, the iHWG was purged with nitrogen. Interferogram were recorded in a wavelength window of $400-4000 \mathrm{~cm}^{-1}$ averaging 30 spectra at a spectral resolution of $2 \mathrm{~cm}^{-1}$ using a Blackman-Harris 3-term apodization function.

\section{Results and discussion}

The characteristics of the riQCL in terms of optical emission power $v s$. applied voltage and current density are shown in Fig. 3. The threshold current density is $4 \mathrm{kA} \mathrm{cm}^{-2}$ with a resulting optical output power of $5 \mathrm{~mW}$ (@ $5.5 \mathrm{kA} \mathrm{cm}^{-2}$ ), and $80 \mathrm{~mW}$ (@10 kA cm ${ }^{-2}$ ), respectively.

As the signal-to-noise ratio and background signal are dependent on the laser driving current, the SNR varied accord-

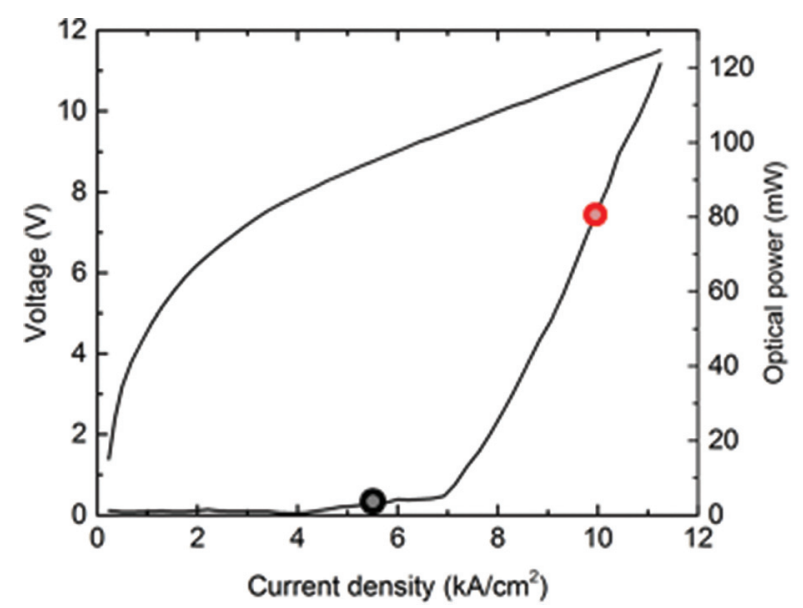

Fig. 3 Voltage vs. current density curve of the riQCL representing the emitted optical output power operated at $293 \mathrm{~K}$ (room temperature) driving the riQCL either at $5.5 \mathrm{kA} \mathrm{cm}^{-2}$ (0: emission wavelength a $1144 \mathrm{~cm}^{-1}$ ) or at $10 \mathrm{kA} \mathrm{cm}^{-2}$ (o: emission wavelength a $1144 \mathrm{~cm}^{-1}$ and $1170 \mathrm{~cm}^{-1}$ ). 
ingly from 344 for a driving current density of $5.5 \mathrm{kA} \mathrm{cm}^{-2}$ to 1951 for $10 \mathrm{kA} \mathrm{cm}^{-2}$.

In order to verify suitable spectral overlap between both riQCL emission lines (i.e., @ 1144 and $1170 \mathrm{~cm}^{-1}$ ) and characteristic absorption bands of furan and 2-methoxyethanol, individual IR spectra of each gas phase constituent were recorded using an FT-IR spectrometer coupled to a suitable iHWG. The gas cell was filled with pure vapor of either furan or 2-methoxyethanol. Likewise, the FT-IR spectrum of the riQCL was recorded by coupling the emitted radiation in lieu of the broadband emitter of the spectrometer into the interferometer of the FT-IR spectrometer, thereby obtaining the emission characteristics of the riQCL. An emission wavelength of $1144 \mathrm{~cm}^{-1}$ was obtained by applying $5.5 \mathrm{kA} \mathrm{cm}^{-2}$ to the riQCL, whereas $10 \mathrm{kA} \mathrm{cm}^{-2}$ led to the emission of both wavelengths (i.e., $1144 \mathrm{~cm}^{-1}$ and $1170 \mathrm{~cm}^{-1}$ ). The IR spectrum of laser emission characteristics (black) are overlaid with the spectral signatures of furan (red) and 2-methoxyethanol (blue) in Fig. 4.

Additional FT-IR spectroscopic studies of various furan and 2-methoxyethanol mixtures in a concentration range of $0-100 \%$ indicate distinctly changing spectral features, as shown in Fig. 5. After purging the iHWG with approx. $50 \mathrm{~mL}$ of gas mixture, the inlet/outlet gas ports of the iHWG were closed off via a T-valve, and 30 spectral scans were averaged.

The same experimental procedure was then applied for characterizing the performance and multicomponent detection capabilities of the riQCL-iHWG sensor system.

Fig. 6A shows the riQCL-iHWG sensor response as a function of increasing furan concentrations (2-20\% in 2-methoxyethanol) at an applied laser voltage of $10 \mathrm{kA} \mathrm{cm}^{-2}$, at which both wavelengths (i.e., $1144 \mathrm{~cm}^{-1}$ and $1170 \mathrm{~cm}^{-1}$ ) are emitted. As the absorption of furan overlaps with the laser emission at $1170 \mathrm{~cm}^{-1}$, it is immediately evident that increasing concentrations of furan accordingly reduced the detected signal. The obtained response function is fitted with an exponential

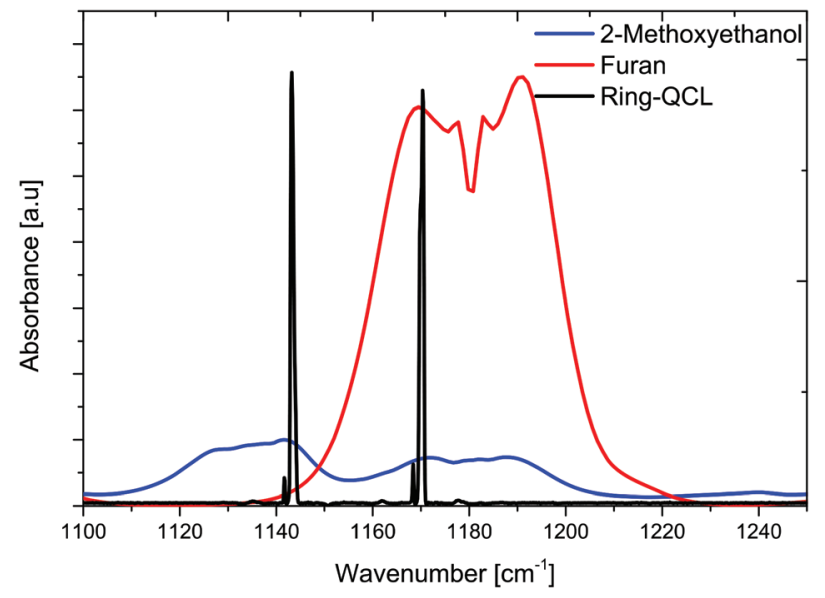

Fig. 4 Laser emission lines (black) overlaid with the IR spectrum of furan (red), and 2-methoxyethanol (blue). The emission spectrum of the riQCL was obtained by coupling the emitted laser radiation into a Fourier transform infrared (FT-IR) spectrometer.

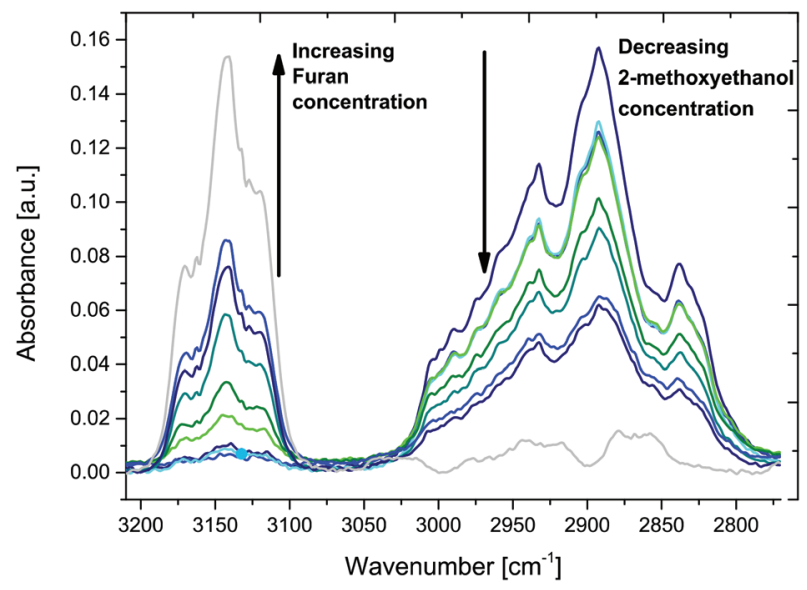

Fig. 5 For comparison, IR spectra were recorded by coupling an iHWG with $7.5 \mathrm{~cm}$ channel length to a conventional FT-IR spectrometer analyzing vapor phase samples with increasing concentrations of furan, and correspondingly decreasing concentrations of 2-methoxyethanol (0 to $100 \%$ for each constituent).

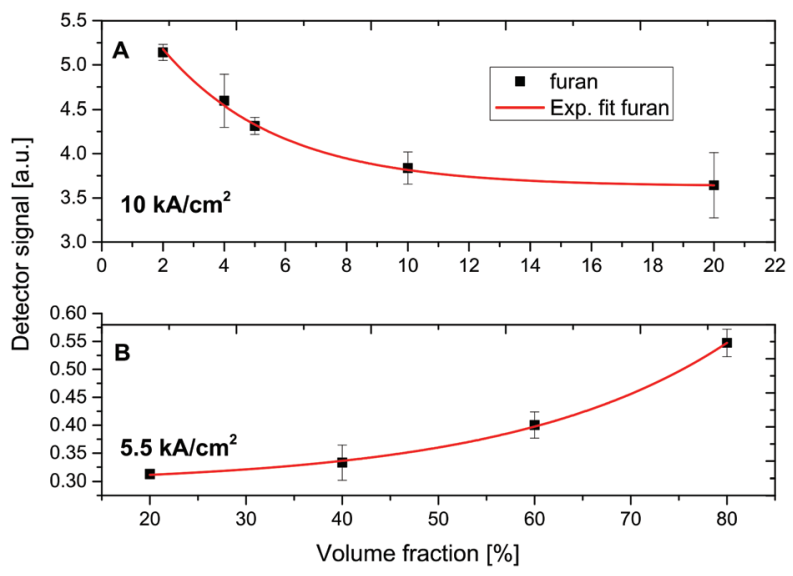

Fig. 6 riQCL-iHWG sensor system response to furan (in 2-methoxyethanol) obtained via selective emission line damping at an applied voltage of $10 \mathrm{kA} \mathrm{cm}^{-2}(\mathrm{~A})$, and of $5.5 \mathrm{kA} \mathrm{cm}^{-2}$ (B). An exponential fit was applied to the response function resulting in a calibration model with a coefficient of determination $\left(R^{2}\right)>0.99$ for both curves.

curve, thereby resulting in a calibration function with a coefficient of determination $\left(R^{2}\right)$ value $>0.99$.

Using the same procedure, the furan fractions were increased (20-80\% in 2-methoxyethanol), and introduced into the iHWG. However, now the laser emission was tuned to $1144 \mathrm{~cm}^{-1}$ at $5.5 \mathrm{kA} \mathrm{cm}^{-2}$ of voltage applied to the riQCL, thus matching the absorption of 2-methoxyethanol (Fig. 6B).

Due to the decreasing concentrations of 2-methoxyethanol in the gas cell, a decreased in damping of the radiation emitted from the QCL readily verified that the narrow frequency band emitted at $1144 \mathrm{~cm}^{-1}$ overlapped with the absorption feature characteristic. The positive slope of the exponential fit $\left(R^{2}>0.99\right)$ reveals the expected behavior. 
During these experiments, the riQCL-iHWG sensor system was operated at a repetition rate of $10 \mathrm{kHz}$ with a pulse duration of $100 \mathrm{~ns}$, whereby emission was achieved at room temperature for an applied voltage of $10 \mathrm{kA} \mathrm{cm}^{-2}$. Similar characteristics were ensured at an applied voltage of $5.5 \mathrm{kA} \mathrm{cm}^{-2}$ with a pulse duration of $250 \mathrm{~ns}$ at the same repetition rate.

The magnitude of the error bars shown for the experimental data points the calibration functions (Fig. 6) result predominantly from the manual gas mixing procedures, however suffice to demonstrate the analytical utility of the developed riQCL-iHWG sensor prototype in multicomponent vapor phase sensing scenarios.

\section{Conclusions and outlook}

In this study, we demonstrated the first combination of substrate-integrated hollow waveguides with vertically emitting ring-shaped two-color quantum cascade lasers for gas/vapor phase sensing applications. Specifically, the developed riQCLiHWG sensor prototype comprises a riQCL with an embossed $2^{\text {nd }}$ order DFB grating emitting at $1144 \mathrm{~cm}^{-1}$ and $1170 \mathrm{~cm}^{-1}$ at room temperature in pulsed mode, and a miniaturized iHWG gas cell simultaneously serving as MIR waveguide with a sample volume of only $600 \mu \mathrm{L}$.

By directly coupling the laser emission into the iHWG, two exemplary analytes - furan and 2-methoxyethanol - were simultaneously detected and quantified during proof-of-principle analytical studies. This innovative sensing concept not only promises exceptionally compact mid-infrared gas sensing platforms, but efficient quantitative vapor phase analysis in minute (i.e., few hundreds of microliters) sample volumes in sensing/ monitoring scenarios requiring rapid measurements (i.e., few seconds) due to a transiently changing sample composition.

In a next step, a significant further reduction of the device footprint is anticipated by direct integration of the riQCL and a thermoelectrically cooled MCT detector or a pyroelectric detection device into the iHWG structure. Such hybrid integration not only ensures more efficient optical energy throughput, but also avoids usage of any additional beam guiding optics, while maintaining or even improving the device robustness (e.g., changes in optical alignment due to mechanical vibrations, etc.). Consequently, the application of such devices in sensing applications including environmental analysis, process monitoring, security and surveillance scenarios, and biomedical diagnostics appears a natural fit.

\section{Acknowledgements}

Partial support of this study by the project APOSEMA funded by the German BMBF within the M-Era.net program is greatly acknowledged. The Machine Shop at Ulm University is thanked for support during prototype development of the iHWG. This work was performed in part under the auspices of the U.S. Department of Energy by Lawrence Livermore National Laboratory
(LLNL) under Contract DE-AC52-07NA27344. This project was funded in part under LLNL sub-contract No. B603018 and B607114. This work was also supported by the Austrian Science Fund (FWF) via project Next-Lite (F49-P09).

\section{Notes and references}

1 B. Mizaikoff, Chem. Soc. Rev., 2013, 42, 8683.

2 M. Sieger, J. Haas, M. Jetter, P. Michler, M. Godejohann and B. Mizaikoff, Anal. Chem., 2016, 88, 2558-2562.

3 J. Haas and B. Mizaikoff, Annu. Rev. Anal. Chem., 2016, 9(1), 45-68.

4 B. Mizaikoff, Anal. Chem., 2003, 75, 258 A-267 A.

5 J. Faist, F. Capasso, D. L. Sivco, C. Sirtori, A. L. Hutchinson and A. Y. Cho, Science, 1994, 264, 553-556.

6 K. Wörle, F. Seichter, A. Wilk, C. Armacost, T. Day, M. Godejohann, U. Wachter, J. Vogt, P. Radermacher and B. Mizaikoff, Anal. Chem., 2013, 85, 2697-2702.

7 C. Charlton, F. de Melas, A. Inberg, N. Croitoru and B. Mizaikoff, IEE Proc.: Optoelectron., 2003, 150, 306.

8 C. Charlton, A. Katzir and B. Mizaikoff, Anal. Chem., 2005, 77, 4398-4403.

9 E. Mujagić, L. K. Hoffmann, S. Schartner, M. Nobile, W. Schrenk, M. P. Semtsiv, M. Wienold, W. T. Masselink and G. Strasser, Appl. Phys. Lett., 2008, 93, 161101.

10 E. Mujagić, S. Schartner, L. K. Hoffmann, W. Schrenk, M. P. Semtsiv, M. Wienold, W. T. Masselink and G. Strasser, Appl. Phys. Lett., 2008, 93, 011108.

11 E. Mujagić, C. Deutsch, H. Detz, P. Klang, M. Nobile, A. M. Andrews, W. Schrenk, K. Unterrainer and G. Strasser, Appl. Phys. Lett., 2009, 95, 011120.

12 Y. Bai, S. Tsao, N. Bandyopadhyay, S. Slivken, Q. Y. Lu, D. Caffey, M. Pushkarsky, T. Day and M. Razeghi, Appl. Phys. Lett., 2011, 99, 261104.

13 C. Schwarzer, R. Szedlak, S. Il Ahn, T. Zederbauer, H. Detz, A. Maxwell Andrews, W. Schrenk and G. Strasser, Appl. Phys. Lett., 2013, 103, 081101.

14 R. Szedlak, C. Schwarzer, T. Zederbauer, H. Detz, A. M. Andrews, W. Schrenk and G. Strasser, Opt. Express, 2014, 22, 15829.

15 R. Szedlak, C. Schwarzer, T. Zederbauer, H. Detz, A. Maxwell Andrews, W. Schrenk and G. Strasser, Appl. Phys. Lett., 2014, 104, 151105.

16 R. Szedlak, M. Holzbauer, D. MacFarland, T. Zederbauer, H. Detz, A. M. Andrews, C. Schwarzer, W. Schrenk and G. Strasser, Sci. Rep., 2015, 5, 16668.

17 A. Harrer, R. Szedlak, B. Schwarz, H. Moser, T. Zederbauer, D. MacFarland, H. Detz, A. M. Andrews, W. Schrenk, B. Lendl and G. Strasser, Sci. Rep., 2016, 6, 21795.

18 R. Szedlak, M. Holzbauer, P. Reininger, D. MacFarland, T. Zederbauer, H. Detz, A. M. Andrews, W. Schrenk and G. Strasser, Vib. Spectrosc., 2016, 84, 101-105.

19 A. Wilk, J. C. Carter, M. Chrisp, A. M. Manuel, P. Mirkarimi, J. B. Alameda and B. Mizaikoff, Anal. Chem., 2013, 85, 11205-11210. 
20 J. F. da S. Petruci, P. R. Fortes, V. Kokoric, A. Wilk, I. M. Raimundo, A. A. Cardoso and B. Mizaikoff, Analyst, 2013, 139, 198-203.

21 V. Kokoric, A. Wilk and B. Mizaikoff, Anal. Methods, 2015, 7, 3664-3667.

22 J. F. da S. Petruci, A. A. Cardoso, A. Wilk, V. Kokoric and B. Mizaikoff, Anal. Chem., 2015, 87, 9580-9583.
23 J. F. da S. Petruci, A. Wilk, A. A. Cardoso and B. Mizaikoff, Anal. Chem., 2015, 87, 9605-9611.

24 E. Tütüncü, M. Nägele, P. Fuchs, M. Fischer and B. Mizaikoff, ACS Sens., 2016, 1(7), 847-851.

25 I. José Gomes da Silva, E. Tütüncü, M. Nägele, P. Fuchs, M. Fischer, I. M. Raimundo and B. Mizaikoff, Analyst, 2016, 141, 4432-4437. 